\title{
The Effects of TPL2 Inhibitor Treatments on the Growth of Breast Cancer Cells
}

\author{
Jun-Han Lee ${ }^{1}$ and Joseph H. Jeong ${ }^{2 *}$ \\ ${ }^{1}$ Department of Otorhinolaryngology, Head and Neck Surgery, Medical Research Institute, Kangbuk Samsung Hospital, Sungkyunkwan University \\ School of Medicine, Seoul, Republic of Korea, 03181. \\ ${ }^{2}$ Laboratory of Developmental Biology and Genomics, College of Veterinary Medicine, Seoul National University and Korea Mouse Phenotyping Center, \\ Seoul, Republic of Korea, 08826.
}

\begin{abstract}
Received: November 14, 2016; Accepted: December 19, 2016; Published: January 16, 2017
*Corresponding author: Joseph H. Jeong, Laboratory of Developmental Biology and Genomics, College of Veterinary Medicine, Seoul National University and Korea Mouse Phenotyping Center, Seoul, Republic of Korea, 08826, Tel: 82-02-885-8397; Fax: 82-02-885-8397; E-mail: jeongjoseph@snu.ac.kr
\end{abstract}

\begin{abstract}
Approximately $40 \%$ of human breast cancer show increased TPL2 expression mainly due to TPL2 gene amplification. In this study, we assessed the effects of a small-molecule chemical inhibitor of TPL2 and a previously known natural compound inhibitor of TPL2, luteolin, on breast cancer cell growth. For this purpose, cell growth of two commonly used and well-characterized breast cancer cell lines, MCF7 and MDA-MB-231, was measured by MTT assay with inhibitor treatments. In addition, the effects of the treatments on TPL2 downstream signaling pathways were analyzed by western blot. MCF7 cells were more sensitive to the treatment of either the smallmolecule TPL2 inhibitor or luteolin than MDA-MB-231 cells. Despite inhibition of the cell growth of MCF7 cells with luteolin treatment, we could not detect any inhibition of TPL2 downstream signaling pathways. These results suggest that MCF7 cells are dependent on TPL2 kinase activity for their cell growth. In addition, luteolinmediated MCF7 cell growth inhibition may be not a result of the direct inhibition of TPL2 kinase activity.
\end{abstract}

Keywords: TPL2; Luteolin; Breast cancer

\section{Introduction}

Breast cancer is the most common cancer in women worldwide. About 1 out of 8 women will develop breast cancer during their lifetime in the United

States alone and approximately 3 million breast cancer survivors have gone through treatment [1]. Despite its significance, breast cancer research has been greatly hampered by the highly heterogeneous presentation of human breast cancer with at least 17 distinctive histological subtypes by the WHO classification [2]. Each subtype has unique clinopathological characteristics. Accumulating evidence indicates that breast cancer heterogeneity results from a population of cancer stem cell [3].

Intensive research have identified many genetic alterations associated with breast cancer, including the inherited germline mutations in BRCA1 and BRCA2 genes $[4,5]$ and the acquired somatic mutations or gene copy number amplification in the HER2/neu gene [6]. In addition, over expression of the tumor progression locus 2 (TPL2) gene at the mRNA level was also detected in approximately $40 \%$ of human breast cancer specimens [7].

TPL2, also known as mitogen-activated protein kinase 8 (MAP3K8) and cancer Osaka thyroid (Cot), is a serine/threonine kinase, that activates its downstream signaling pathways ERK, JNK, p38, and NF- $\mathrm{KB}$ in both stimulus- and cell type-specific manners [8]. Functionally, TPL2 is mainly associated with immune responses and tumorigenesis [8, 9]. Notably, TPL2 appears to have a dual role in tumorigenesis, functioning either as a tumor suppressor or as a tumor promoter in a tumor-type specific manner. In breast cancer, several studies indicate that TPL2 promotes tumorigenesis through distinct underlying molecular mechanisms [10-12].

Based on the role of TPL2 as a tumor promoter in breast cancer tumorigenesis, we assessed the effects of TPL2 inhibitors on the cell growth of two human breast cancer cell lines, MCF7 and MDA-MB-231. We also included luteolin, a type of flavonoid that is known to be a natural inhibitor of TPL2.

Here, we show that MCF7 cells are more sensitive to the treatment of either a TPL2 inhibitor treatment or luteolin than MDA-MB-231 cells. Surprisingly, the underlying molecular mechanisms for luteolin-mediated MCF7 cell growth inhibition may be not through the direct inhibition of TPL2 kinase activity.

\section{Materials and Methods}

\section{Reagents}

TPL2 kinase inhibitor (\#616373) was purchased from Calbiochem, and luteolin (\#RRL02) was purchased from Biotang. Antibodies against phospho-Stat3 (Ser727, \#9134), phosphop70S6K (Thr389, \#9205), p70S6K (\#9202), phospho-ERK1/2 (Thr202/Tyr204, \#4370), ERK1/2 (\#4695), phospho-SAPK/ JNK (Thr183/Tyr185, \#4668), and SAPK/JNK (\#9252) were purchased from Cell Signaling Technology. Antibodies against Stat3 (\#sc-482) and $\beta$-actin (\#sc-47778) were purchased from Santa Cruz Biotechnonlogy. 


\section{Cell lines}

Human breast cancer cells (MCF-7 and MDA-MB-231) were kindly provided by Dr. Chengyu Liang (USC) and were maintained in DMEM media (Invitrogen) supplemented with 10\% FBS (Invitrogen), Penicillin-Streptomycin $(100 \mathrm{U} / \mathrm{ml}$ and $100 \mathrm{ug} / \mathrm{ml}$, respectively, Invitrogen), $2 \mathrm{mM}$ L-glutamine (Invitrogen), and $1 \mathrm{mM}$ sodium pyruvate (Invitrogen). Both cell lines were cultured at $37^{\circ} \mathrm{C}$ and humidified $5 \% \mathrm{CO}_{2}$.

\section{Cell growth assay (MTS assay)}

The effect of treatment with either with a TPL2 kinase inhibitor or luteolin on breast cancer cell growth was measured by the CellTiter $96^{\circledR}$ AQueous One Solution Cell Proliferation Assay Kit (Promega). Briefly, MCF-7 and MDA-MB 231 cells (5 x $10^{3}$ cells per well) were seeded into 96-well plates (TPP) and treated with varying concentrations of the TPL2 inhibitor $(0-50$ $\mu \mathrm{M})$ or luteolin $(0-160 \mu \mathrm{M})$. After 48 and 72 hours treatment, 20 $\mu \mathrm{l}$ of the CellTiter $96^{\circledR}$ AQueous One Solution reagent was added to each well and cells were incubated at $37^{\circ} \mathrm{C}$ for 1 hour. The absorbance was measured at $490 \mathrm{~nm}$ using the iMarkTM Micro Plate Reader (Bio-Rad). LC $^{50}$ values (the lethal concentration required to kill $50 \%$ of the cells) were determined by the best-fit curve method.

\section{Western blot}

MCF-7 and MDA-MB-231 cells were treated with the indicated concentrations of a TPL2 kinase inhibitor or luteolin for 24 hours. Cells were lysed with RIPA buffer (Millipore) with complete protease inhibitor cocktail (Roche) and phosphatase inhibitor (Sigma). Then, the protein concentration was measured by the Pierce BCA Protein Assay Kit (Thermo Scientific). $20 \mu \mathrm{g}$ of protein for each sample was separated by SDS-PAGE and transferred to PVDF membranes (Bio-Rad). After blocking with 5
\% nonfat dry milk (Bio-Rad) in TBST (10 mM Tris, $150 \mathrm{mM} \mathrm{NaCl}$, $0.1 \%$ Tween-20, pH 7.4) for 1 hour at room temperature (RT), the membranes were incubated with primary antibodies at $4^{\circ} \mathrm{C}$ overnight. Then, the membranes were washed with TBST three times for $30 \mathrm{~min}$ and incubated with HRP-conjugated secondary antibodies for 1 hour at RT.

After washing three times, proteins were detected with the Pierce ECL Western blotting substrate (Thermo Scientific). Densitometry analysis was performed using Image J software (NIH).

\section{Results \\ Effects of a pharmaceutical small-molecule TPL2 kinase inhibitor on the growth of two breast cancer cell lines, MCF7 and MDA-MB-231.}

To measure the effects of a pharmaceutical small-molecule TPL2 inhibitor on the growth of two breast cancer cell lines, MCF7 and MDA-MB-231, MTS ([3-(4,5-dimethylthiazol-2-yl)-5(3-carboxymethoxyphenyl)-2-(4-sulfophenyl)-2H-tetrazolium, inner salt] assays were performed in triplicate using increasing concentrations of a TPL2 inhibitor for 48 hours and 72 hours. Then, $\mathrm{LC}^{50}$ values (the lethal concentration required to kill $50 \%$ of the cells) were determined using the best-fit curve method. $\mathrm{LC}^{50}$ values for MCF7 cells were $35 \mu \mathrm{M}$ and $20 \mu \mathrm{M}$ for 48 hours and 72 hours, respectively (Fig 1A). LC ${ }^{50}$ values for MDA-MB-231 cells were $163 \mu \mathrm{M}$ for 48 hours and $51 \mu \mathrm{M}$ for 72 hours (Fig 1B). LC $C^{50}$ values for MCF7 cells are significantly lower than those for MDAMB-231 cells for both 48 hours ( $p=0.0011$, t-test) and 72 hours ( $p=0.0007$, t-test). Therefore, MCF7 cells are more responsive to a TPL2 inhibitor than MDA-MB-231 cells.

To investigate the effects of TPL2 inhibitor treatment on TPL2 downstream signaling pathways, we performed western blot
A

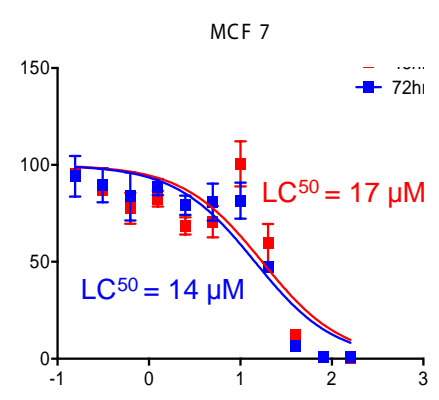

B

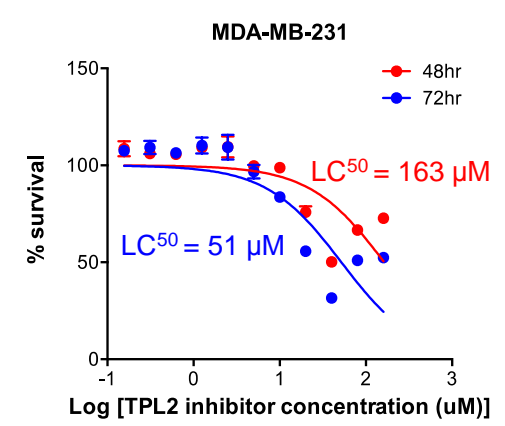

C

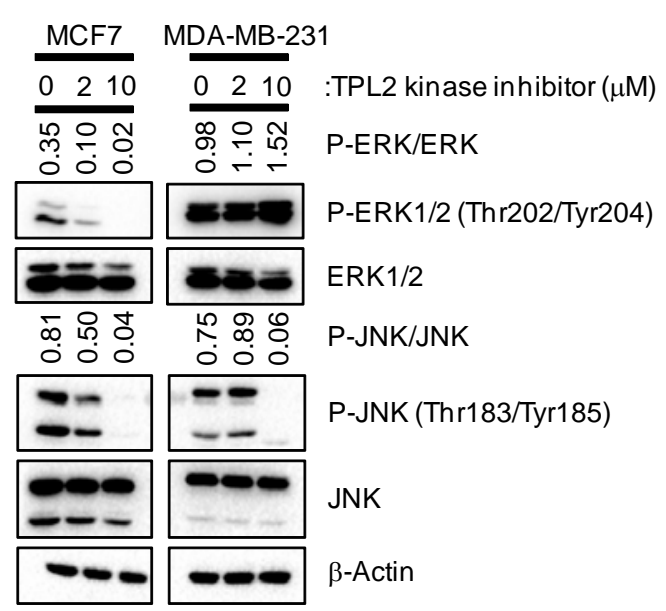

Figure 1: Effects of a TPL2 kinase inhibitor treatment on the growth of both MCF7 cells (A) and MDA-MB-231 cells (B). LC50 values (the lethal concentration required to kill $50 \%$ of the cells) either 48 or 72 hours after TPL2 inhibitor treatment were determined using the MTS assay (A and B). Effects of a TPL2 kinase inhibitor treatment for 24 hours with the indicated concentrations on TPL2 downstream signaling molecules were also analyzed by western blot (C). 
analyses. Treatment of MCF7 cells with a TPL2 inhibitor in two different concentrations below the identified $\mathrm{LC}^{50}(2 \mu \mathrm{M}$ and 10 $\mu \mathrm{M})$ suppressed both the MEK/ERK MAPK pathway as evaluated by the measurement of phospho-ERK (Thr202/Tyr204), and the JNK MAPK pathway as evaluated by the measurement of phosphoJNK (Thr183/Tyr185) (Fig 1C). However, in MDA-MB-231 cells, although there was a suppression of the JNK MAPK pathway with the higher $10 \mu \mathrm{M}$ concentration, we could not observe any suppression of the MEK/ERK MAPK pathway (Fig 1C).

The other TPL2 downstream signaling pathways, including the p38 MAPK and the NF-kappa B pathways, showed no changes with the treatments (data not shown). Therefore, these data suggest that TPL2 inhibitor treatment specifically suppress the MEK/ERK MAPK pathway in MCF7 cells, ultimately resulting in more significantly decreased tumor cell growth in MCF7 cells than MDA-MB-231 cells.

\section{Effects of luteolin, a known natural TPL2 inhibitor on the growth of MCF7 and MDA-MB-231.}

Luteolin, a type of flavonoid, is known to be a natural inhibitor of TPL2. To assess the efficacy of luteolin treatment in inhibiting the tumor cell growth of MCF7 and MDA-MB-231 cells, MTS assays were performed using increasing concentrations of luteolin, and $\mathrm{LC}^{50}$ values with luteolin treatment were determined as above. $\mathrm{LC}^{50}$ values for MCF7 cells were $25 \mu \mathrm{M}$ and $11 \mu \mathrm{M}$ for 48 hours and 72 hours, respectively (Fig 2A). LC ${ }^{50}$ values for MDAMB-231 cells were $366 \mu \mathrm{M}$ for 48 hours and $163 \mu \mathrm{M}$ for 72 hours (Fig 2B). LC ${ }^{50}$ values with luteolin treatment for MCF7 cells are also significantly lower than those for MDA-MB-231 cells for both 48 hours ( $p=0.0015$, t-test) and 72 hours ( $p=0.0004$, $t$-test $)$. Therefore, MCF7 cells are also more sensitive to a TPL2 inhibitor treatment than MDA-MB-231 cells.

To investigate the effects of luteolin treatment on TPL2 downstream signaling pathways, we performed western blot analyses as above. To our surprise, treatments with luteolin in two different concentrations $(5 \mu \mathrm{M}$ and $20 \mu \mathrm{M})$ could not suppress both the MEK/ERK MAPK pathway and the JNK MAPK pathway in both MCF7 and MDA-MB-231 cells (Fig 2C). Therefore, these data demonstrate that luteolin treatment could not suppress any TPL2 downstream signaling pathway in MCF7 cells despite decreasing tumor cell growth. These results suggest that the underlying molecular mechanism for luteolin-mediated MCF7 cell growth inhibition is not through the direct inhibition of TPL2 kinase activity.

Effects of a pharmaceutical small-molecule TPL2 inhibitor and luteolin on STAT3 and mTOR signaling pathways.

To gain an insight into the molecular mechanisms underlying the differential responses to a TPL2 inhibitor and luteolin between MCF7 and MDA-MB-231 cells, we assessed two other signaling pathways, STAT3 and mTOR, which are important for breast cancer development and progression. Treatment with a TPL2 kinase inhibitor suppressed activation of the STAT3 signaling pathway, as evaluated by the measurement of phosphoSTAT3 (Ser727), only in MCF7 cells, but luteolin treatment had no effects on the activation status of STAT3 in both cell lines (Fig 3A and $3 \mathrm{~B}$ ). Therefore, it is possible that the TPL2 kinase inhibitor inhibits MCF7 tumor cell growth through the suppression of the STAT3 signaling pathway. Treatments with a TPL2 kinase inhibitor and luteolin suppressed the mTOR pathway, as evaluated by the measurement of phospho-70S6K (Thr389), in both cell lines (Fig 3A and 3B). Since there were no differences in the activation status of the STAT3 and mTOR signaling pathways between MCF7 and MDA-MB-231 cells with the treatment of luteolin, luteolin likely inhibits MCF7 cell growth through other unknown mechanisms.
A

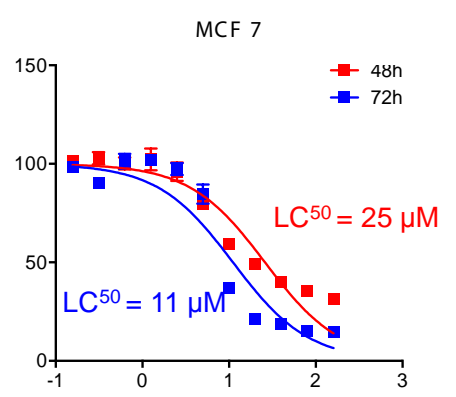

B

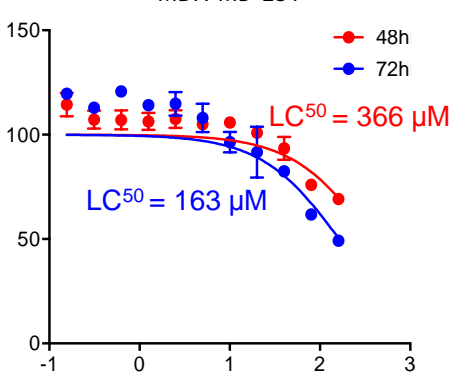

C

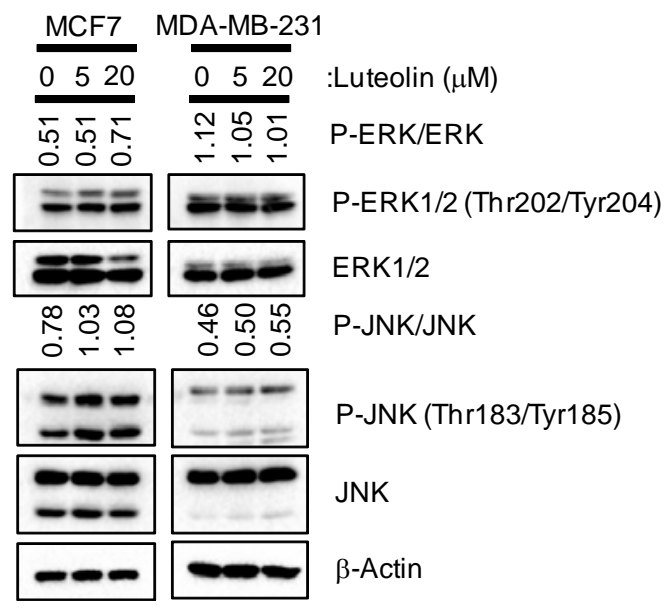

Figure 2: Effects of luteolin treatment on the growth of both MCF7 cells (A) and MDA-MB-231 cells (B). LC ${ }^{50}$ values either 48 or 72 hours after luteolin treatment were determined using the MTS assay (A and B). Effects of a luteolin treatment for 24 hours with the indicated concentrations on TPL2 downstream signaling molecules were also analyzed by western blot (C). 
A

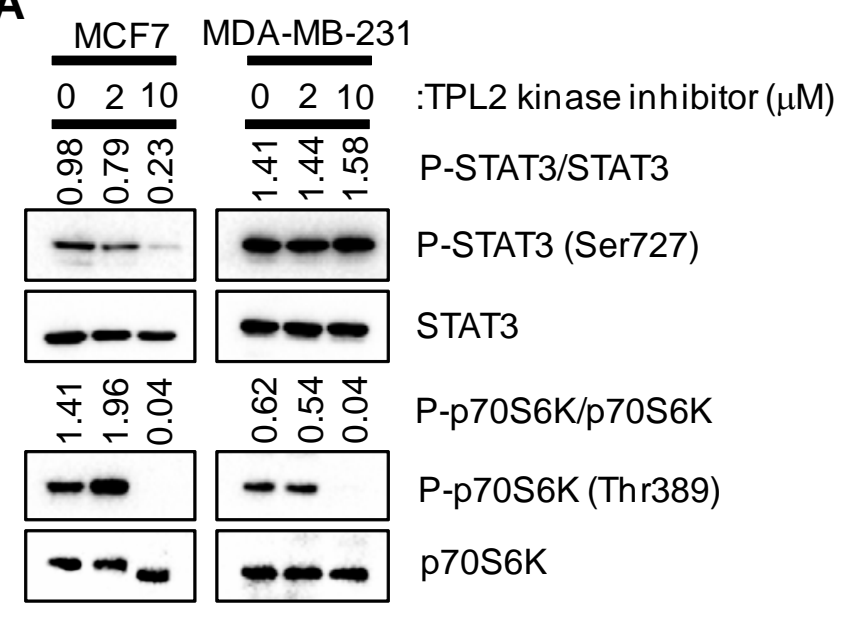

B

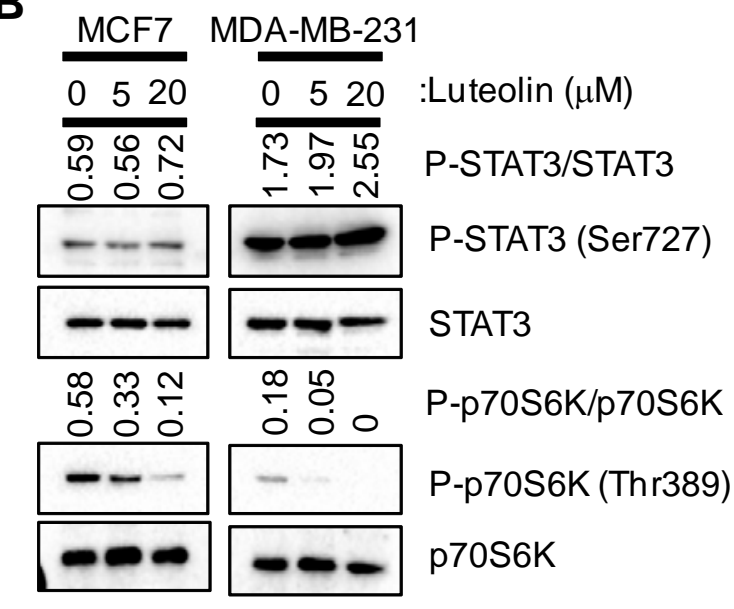

Figure 3: Effects of either treatment with a TPL2 kinase inhibitor or luteolin for 24 hours on the STAT signaling pathway and the mTOR pathway were analyzed in MCF7 cells (A) and MDA-MB-231 cells (B) by western blot.

\section{Discussion}

TPL2 is over expressed in approximately $40 \%$ of human breast cancers. Given that mutations in the TPL2 gene is very rare in human cancers, increased expression of TPL2 through TPL2 gene amplification appears to contribute to breast cancer tumorigenesis. We examined effects of two TPL2 inhibitors, a small-molecule chemical inhibitor of TPL2 and a known natural inhibitor of TPL2, luteolin, on the cell growth of two commonly used and well-characterized breast cancer cell lines, MCF7 and MDA-MB-231. Our data show that treatment with either a TPL2 kinase inhibitor or luteolin more significantly suppressed the cell growth of MCF7 cells than that of MDA-MB-231 cells. Luteolin inhibits each step of human tumorigenesis by targeting many signaling pathways, including JNK, NF- $\mathrm{BB}$, TRAIL, TNF- $\alpha$, p53, Wnt/ $\beta$-catenin, HSP90, mTOR, IGF1, AKT1, etc. In particular, the $\mathrm{ER} \alpha, \mathrm{p} 53$, and mTOR pathways are targeted by luteolin in breast cancer to inhibit cancer cell growth.

However, despite the successful identification of the numerous dysregulated signaling pathways by luteolin, luteolinbinding partners remain unknown. The MCF7 cell line is luminal type, ER-positive, PR-positive, and HER2-negative with wildtype p53 protein, while MDA-MB-231 is basal type, ER-negative, PR-negative, HER2-negative with mutant p53 (R280K) protein. Therefore, based on these genotypes, it is possible that the ER and p53 signaling pathways are associated with the enhanced sensitivity of MCF7 cells to luteolin than MDA-MB-231 cells. To support this possibility, it has been previously shown that luteolin inhibits the growth of MCF7 cells by inhibiting IGF-1 pathways-dependent ER $\alpha$ expression. Although a significant increase in p53 expression with luteolin treatment has been shown to result in apoptotic cell death of MDA-MB-231 cells, the used concentrations were much higher than those used in this study.

Previously, it has been shown that luteolin binds directly to the ATP binding site of TPL2 and inhibits TPL2 kinase activity in an ATP-competitive manner using in vitro TPL2 kinase assay, pull-down assay with luteolin- Sepharose 4B, and computer modeling study. Functionally, the treatment of JB6 P+ cells with luteolin inhibited TNF- $\alpha$ treatment-induced TPL2 kinase activity in the cells, leading to the suppression of TPL2 downstream signaling pathways, including the MEK/ERK MAPK, JNK MAPK, and AKT pathways. However, luteolin treatment did not suppress any TPL2 downstream signaling pathway in MCF7 cells despite its significant inhibitory effect on the cell growth of MCF7 cells. Therefore, the inhibitory effect on the cell growth of MCF7 cells by luteolin is not through the direct inhibition of TPL2 kinase activity.

\section{Acknowledgement}

This research was supported by Korea Mouse Phenotyping Project (2013M3A9D5072550) of the Ministry of Science, ICT and Future Planning through the National Research Foundation.

\section{Conflicts of interest}

No potential conflicts of interest were disclosed.

\section{References}

1. Carol DeSantis, Jiemin Ma, Leah Bryan and Ahmedin Jemal. Breast cancer statistics 2013. CA: a cancer journal for clinicians. 2014;64(1):52-62.

2. Weigelt B, Reis-Filho JS. Histological and molecular types of breast cancer: is there a unifying taxonomy? Nat Rev Clin Oncol. 2009;6(12):718-730. doi: 10.1038/nrclinonc.2009.166.

3. Pece S, Tosoni D, Confalonieri S, Mazzarol G, Vecchi M, Ronzoni S, et al. Biological and molecular heterogeneity of breast cancers correlates with their cancer stem cell content. Cell. 2010;140(1):62-73. doi: 10.1016/j.cell.2009.12.007

4. Hall JM, Lee MK, Newman B, Morrow JE, Anderson LA, Huey B, et al. Linkage of early-onset familial breast cancer to chromosome 17q21. Science. 1990;250(4988):1684-1689. 
5. Miki Y, Swensen J, Shattuck-Eidens D, Futreal PA, Harshman K, Tavtigian $\mathrm{S}$, et al. A strong candidate for the breast and ovarian cancer susceptibility gene BRCA1. Science. 1994;266(5182):66-71.

6. Coussens L, Yang-Feng TL, Liao YC, Chen E, Gray A, McGrath J, et al. Tyrosine kinase receptor with extensive homology to EGF receptor shares chromosomal location with neu oncogene. Science. 1985;230(4730):1132-1139.

7. G Sourvinos, C Tsatsanis, DA Spandidos. Over expression of the Tpl-2/ Cot oncogene in human breast cancer. Oncogene. 1999;18(35):49684973.

8. T Gantke, S Sriskantharajah, SC Ley. Regulation and function of TPL-2, an IkappaB kinase-regulated MAP kinase kinase kinase. Cell research. 2011;21(1):131-145. doi: 10.1038/cr.2010.173.

9. HW Lee, HY Choi, KM Joo, DH Nam. Tumor progression locus 2
(Tpl2) kinase as a novel therapeutic target for cancer: double-sided effects of Tpl2 on cancer. International journal of molecular sciences. 2015;16(3):4471-4491. doi: 10.3390/ijms16034471.

10. Kim G, Khanal P, Kim JY, Yun HJ, Lim SC, Shim JH, et al. COT phosphorylates prolyl-isomerase Pin1 to promote tumorigenesis in breast cancer. Mol Carcinog. 2015;54(6):440-448. doi: 10.1002/ mc.22112.

11. Kim K, Kim G, Kim JY, Yun HJ, Lim SC, Choi HS. Interleukin-22 promotes epithelial cell transformation and breast tumorigenesis via MAP3K8 activation. Carcinogenesis. 2014;35(6):1352-1361. doi: 10.1093/ carcin/bgu044.

12. Kim G, Khanal P, Lim SC, Yun HJ, Ahn SG, Ki SH, et al. Interleukin-17 induces AP-1 activity and cellular transformation via upregulation of tumor progression locus 2 activity. Carcinogenesis. 2013;34(2):341350. doi: 10.1093/carcin/bgs342. 